\title{
Los derechos reproductivos y los hombres. El debate pendiente
}

\author{
Elsa S. Guevara Ruiseñor
}

\begin{abstract}
El reciente auge en los estudios de la masculinidad en México, ha dado como consecuencia que temas claves dentro del discurso feminista como el de los derechos reproductivos sean ahora motivo de reflexión para el caso de los varones; sin embargo, hace falta considerar las coordenadas de un orden genérico que permita identificar cuáles características adquieren estos derechos en los varones en virtud de la diferencia biológica, de las asimetrías de poder con respecto a las mujeres y en función de la posición social de unos y otras en el plano material y simbólico. Si bien en nuestro país el tema apenas empieza a ser debatido, ha faltado un análisis de fondo sobre lo que significa incorporar el tema de los derechos reproductivos al debate sobre las masculinidades y los hombres. Los pocos esfuerzos que existen parten con frecuencia de una concepción restringida de derechos reproductivos, trasladan mecánicamente a los hombres las demandas de las mujeres, no toman en cuenta que los procesos reproductivos se gestan en los cuerpos de las mujeres, omiten el impacto de las posiciones de poder entre hombres y mujeres, plantean una visión voluntarista de los varones y desconocen el papel de las instituciones en estos procesos.
\end{abstract}

The recent boom in studies on masculinity in Mexico has led to key themes in feminist discourse, such as reproduction rights, now becoming a matter for reflection in the case of males. However, it is necessary to consider the coordinates of a generic order which provides the means of identifying the characteristics of these masculine rights by virtue of biological differences, asymmetry of power regarding women, and according to social position of each at the material and symbolic level. Although the topic is just starting to be debated in Mexico, an in-depth analysis is lacking on what it means to incorporate the subject of reproduction rights into the debate on masculinity and men. The few efforts which exist often stem from a restricted conception of reproduction rights, mechanically shifting women's demands to men; they do not take into account the fact that the reproduction processes take place in women's bodies, they omit the impact of the positions of power between men and women. They propose a voluntaristic vision of males and are not aware of the role of institutions in these processes.

ELSA S. GUEVARA: UNAM Zaragoza.

Desacatos, núm. 11, primavera 2003, pp. 105-119.

* El presente trabajo responde al artículo “Varones, reproducción y derechos: ¿podemos combinar estos términos?”, de Juan Guillermo Figueroa Perea, publicado en Desacatos, núm. 6, Sexualidades, 2001. 


\section{INTRODUCCIÓN}

E

1 reciente auge en los estudios de la masculini-

dad en México, ha traído como consecuencia

que temas claves dentro del discurso feminista como el de los derechos reproductivos sean ahora motivo de reflexión para el caso de los varones. Si los derechos reproductivos son parte constitutiva de los derechos humanos, se dice, entonces no se debe hablar sólo de aquellos que competen a las mujeres sino también a los hombres. Esta observación, incuestionable de principio, obliga a considerar la relación entre distintos sujetos de derecho y los dilemas que suponen las prerrogativas de que goza cada uno, pues si bien no existe duda de que en la relación entre los individuos y el Estado, u otras instancias de regulación social como el mercado o la Iglesia, los hombres pueden sustentar sus demandas en los mismos principios que las mujeres, también es necesario considerar las coordenadas de un orden genérico que permita identificar cuáles características adquieren estos derechos en los varones, en virtud de la diferencia biolójeres y en función de la posición social de unos y otras en el plano material y simbólico. Además lleva a pensar cómo es posible ejercerlos sin que esto se constituya en un contrapeso a los derechos de las mujeres, en especial si consideramos que hasta ahora los movimientos proderechos de los hombres en países como Estados Unidos son altamente conservadores, de tal modo que han utilizado el tema de los derechos como una estrategia política para revertir los avances logrados por las mujeres en este ámbito.

En nuestro país el tema apenas empieza a ser debatido, pero ocurre en un contexto marcado por el avance de los grupos conservadores ${ }^{1}$ quienes siempre se han opuesto a

\footnotetext{
${ }^{1}$ Ya no es sólo la jerarquía de la Iglesia católica que excomulga y condena a las mujeres por ejercer sus derechos reproductivos, ahora también los funcionarios de las instituciones de salud, los aparatos judiciales y las cámaras de representantes son importantes obstáculos en el ejercicio de estos derechos. Así ocurrió en el caso de la niña Paulina en Baja California, en Chiapas donde los aparatos judiciales se convirtieron en ejecutores de las denuncias de los grupos Pro Vida para allanar la clínica Mary Stopes, detener a la doctora y la enferme
}

la libre determinación de las personas para decidir sobre sus procesos reproductivos. Sin embargo, recientemente empiezan a utilizar el discurso de los derechos para reclamar la paternidad de los hombres en los casos en que las mujeres decidan abortar o bien para defender el orden patriarcal de la familia, incluso se han planteado campañas de paternidad para contrarrestar las demandas de despenalización del aborto. Así, hablar de los derechos reproductivos de los hombres puede abonar el terreno de la intolerancia si no se aborda el tema con la rigurosidad teórica que requiere y se toma en cuenta el potencial político de estos conceptos. Para ello es necesario abrir el debate sobre los diferentes vínculos presentes en el tema de los derechos reproductivos y los hombres, puesto que éste no es sólo un asunto de inclusión sino que involucra importantes coordenadas éticas y políticas.

Si bien tanto en la academia como en las ONG de hombres y mujeres hay amplias coincidencias respecto a reivindicar el derecho de las mujeres para decidir sobre sus procesos reproductivos, ha faltado un análisis de fondo sobre lo que significa incorporar el tema de los derechos reproductivos al debate sobre las masculinidades y los hombres. Los pocos esfuerzos que existen parten con frecuencia de una concepción restringida de derechos reproductivos, trasladan mecánicamente a los hombres las demandas de las mujeres, no toman en cuenta que los procesos reproductivos se gestan en los cuerpos de las mujeres, omiten el impacto de las posiciones de poder entre hombres y mujeres, plantean una visión voluntarista de los varones, desconocen el papel de las instituciones como la Iglesia, la familia o el mercado para limitar el ejercicio de los derechos reproductivos e ignoran las dimensiones éticas y políticas que subyacen a estos procesos. Además, es frecuente encontrar una confusión respecto al ámbito propio de los derechos reproductivos en los hombres. Así en ocasiones se encuentran diluidos

ra responsables (nota de H. Bellinghausen, 2000, en La Jornada) y en el Distrito Federal donde los representes del PAN se opusieron tajantemente a las modificaciones a la legislación sobre el aborto. El avance de los fundamentalismos es una tendencia a nivel mundial que pretende, como señala María Consuelo Mejía (1999), revertir acuerdos tan importantes como los alcanzados en El Cairo en 1994. Y todo apunta a un endurecimiento cada vez mayor. 
en demandas más amplias sobre salud reproductiva, confundidos con los derechos sexuales, circunscritos a ámbitos muy reducidos como el de la coitalidad o remitidos a espacios que borran de la agenda a las mujeres, como ciertas propuestas sobre paternidad.

Por ello, la reflexión sobre el tema requiere abordar cuando menos tres puntos claves en el debate: por una parte, precisar los alcances y significados del concepto de derechos reproductivos por la otra, destacar la importancia de considerar las prácticas sexuales y reproductivas de los hombres como factores claves para obstaculizar o facilitar el ejercicio de los derechos reproductivos de las mujeres y, finalmente, identificar cuáles son los ejes de análisis que deben tomarse en cuenta cuando se habla de los hombres como titulares de sus propios derechos reproductivos.

\section{LOS DERECHOS REPRODUCTIVOS. LOS PROBLEMAS DE INTERPRETACIÓN}

Precisar conceptualmente lo que significa hablar de derechos reproductivos no es sólo una exigencia académica, es también un recurso fundamental en la arena política donde la disputa por los significados se vuelve parte central de las batallas en los procesos de legitimación. Con frecuencia se vacía de contenido a conceptos claves, se silencia ciertos componentes, se simplifican sus alcances o se les subsume bajo otros conceptos considerados "políticamente más correctos" a fin de limar sus aristas más filosas.

A diferencia del concepto de salud reproductiva que surge en la convergencia de los aparatos institucionales y los movimientos de mujeres, el concepto de derechos reproductivos se ubica en el marco no institucional que inicia en la lucha por el aborto y la anticoncepción en los países desarrollados, cuya primera instancia de legitimación fue un encuentro internacional feminista relativamente marginal realizado en Amsterdam en $1984 .^{2} \mathrm{El}$

2 Si bien la idea de decisión libre y responsable acerca del número y espaciamiento de los hijos fue declarado por primera vez un derecho humano en 1968 y ratificado en 1969, es hasta el Cuarto Encuentro

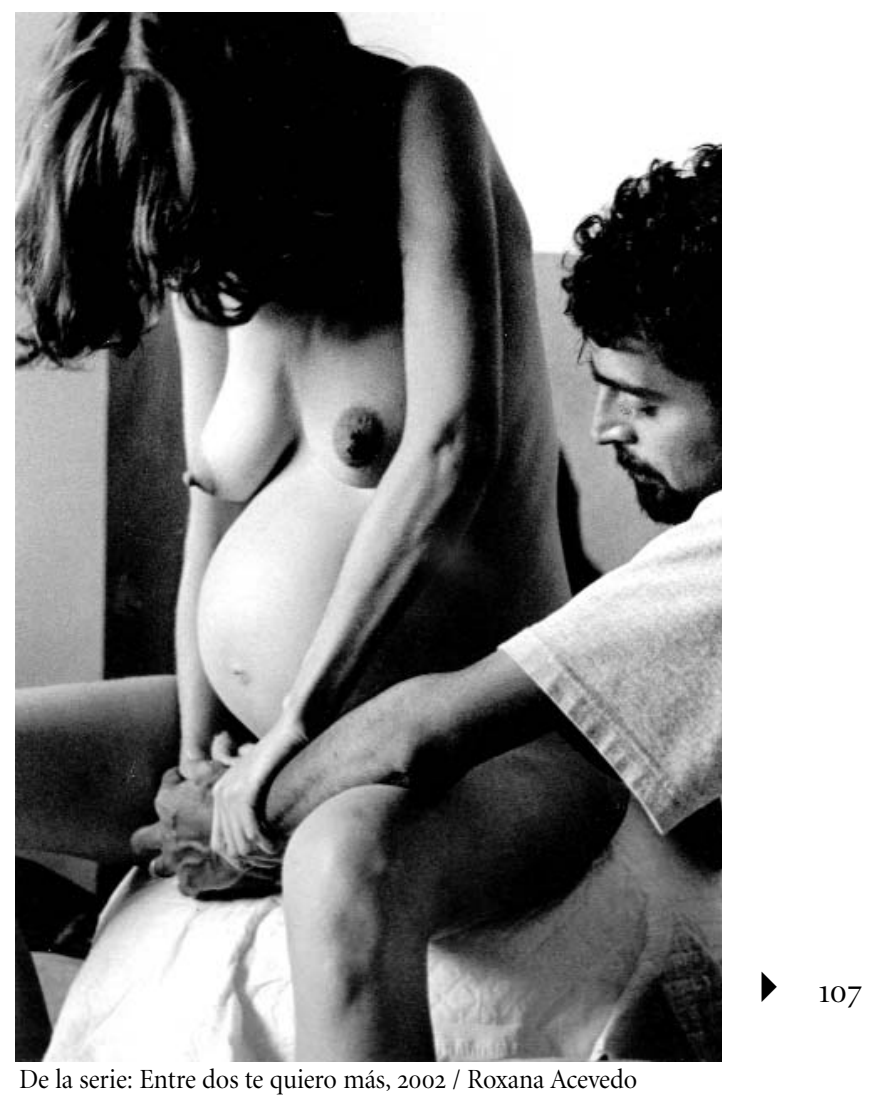

concepto fue refinado en colaboración con activistas e investigadores en el campo de los derechos humanos en los siguientes años, legitimado en Viena en 1993 y retomado en las conferencias subsecuentes de las Naciones Unidas. Sin embargo, las ideas de integridad corporal y autodeterminación sexual datan de 1830 cuando en círculos feministas y socialistas en Inglaterra se defendía el reconocimiento de las mujeres como agentes morales con proyectos y objetivos propios. El potencial político de este concepto se encuentra en su carácter subversivo

Internacional de Mujeres y Salud que el concepto de derechos reproductivos es utilizado por primera vez. Ahí, la International Contraception, Abortion and Sterilisation Campaign, organizadora del evento, se transformó en la Women's Global Network for Reproductive Rights y el lenguaje de los derechos reproductivos ingresó así al movimiento internacional de mujeres (Durand y Gutiérrez, 1998). 
respecto a las asimetrías de poder, presentes tanto en las relaciones entre individuos como entre individuos e instituciones, puesto que tener derechos presume siempre un reequilibrio en las relaciones de poder y un horizonte que apunta hacia la justicia y el empoderamiento (Correa, 1996).

En México el concepto de derechos reproductivos es reciente, pero las premisas que dan contenido a este concepto datan de mucho tiempo atrás cuando las mujeres empiezan a reclamar derechos civiles y políticos que les permitieran tomar decisiones sobre sus procesos reproductivos al considerar que en ello estaba en juego su salud y su vida. La mortalidad materna, la morbilidad y daños generados por los abortos clandestinos y los embarazos no deseados en adolescentes, fueron en las décadas de los veinte y los treinta los argumentos centrales de los movimientos feministas y de izquierda para que el Estado reconociera el peso que tienen los procesos reproductivos en la salud de las mujeres. De igual manera, las demandas por el acceso a los métodos anticonceptivos y la despenalización del aborto en la década de los setenta estaban ligadas a la idea de integridad corporal y libre autodeterminación. Cuando las mujeres exigían "anticonceptivos para no abortar", "aborto gratuito para no morir" y reclamaban la maternidad libre y voluntaria, lo hacían considerando el derecho de las mujeres a no sufrir daños a su salud ni arriesgar su vida para que la maternidad fuera una opción y no un destino, pero también estaba presente el rechazo a los embarazos impuestos mediante la violencia física y sexual que vivían las mujeres en su vida cotidiana y a las políticas poblacionistas que hacían de la maternidad la única forma de reconocimiento de las mujeres.

Con la consolidación de las políticas públicas orientadas a regular la fertilidad en la década de los ochenta, nuevamente el sentido de la libre determinación e integridad corporal cobran vida en las denuncias sobre esterilizaciones forzadas, en el rechazo a métodos de planificación familiar lesivos a la salud y en las políticas de población que no tomaban en cuenta las necesidades e

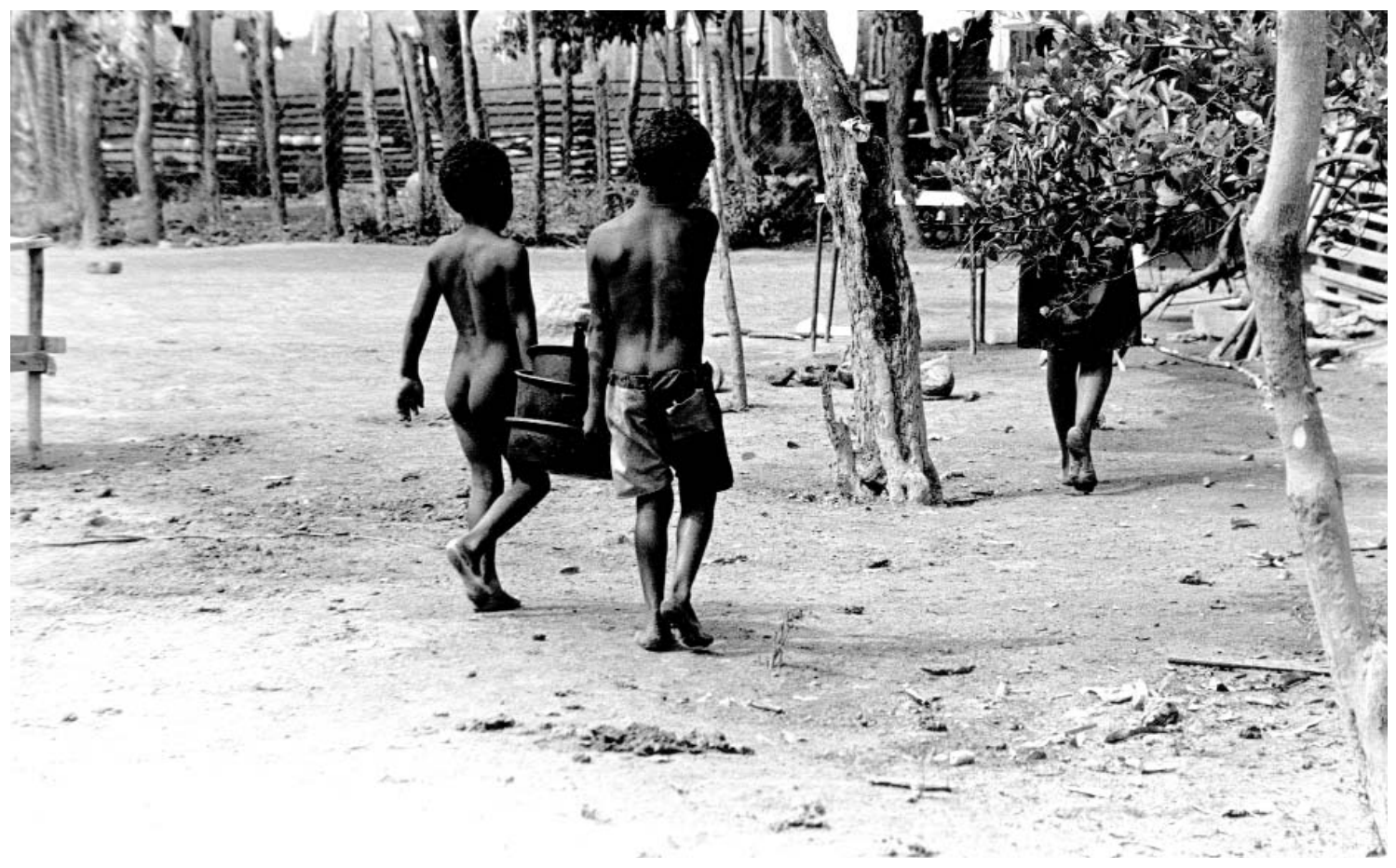

Costa Chica, Oaxaca, 2000 / Roxana Acevedo 
intereses de las mujeres. Finalmente, en 1994 en la Conferencia Internacional sobre Población y Desarrollo, los derechos reproductivos se legitiman institucionalmente y el concepto se extiende más allá de los círculos feministas. Se reconoce el derecho de los individuos y las parejas de decidir de manera libre y responsable sobre el número y espaciamiento de sus hijos y se acepta la libertad de elección en el marco más amplio del ejercicio de la ciudadanía y los derechos humanos. Sin embargo, esta institucionalización ${ }^{3}$ significó también una despolitización del concepto, hizo a un lado sus componentes más radicales y redujo su contenido a la decisión de cómo y cuándo tener hijos/as.

En realidad su contenido ético es bastante más amplio y profundo, Correa y Petchesky (1994) señalan cuatro principios fundamentales: integridad corporal, autodeterminación, igualdad y diversidad. Integridad corporal incluye el derecho de las personas a no ser alienadas de su capacidad sexual y reproductiva, a mantener la integridad física de su persona; al mismo tiempo, implica derechos afirmativos para disfrutar plenamente de su cuerpo. La autodeterminación se refiere a la capacidad para decidir de manera libre e informada sobre el ejercicio de su sexualidad y sus opciones reproductivas; implica el respeto por la manera en que las personas toman decisiones, los valores que tienen peso para ellas/ellos y las redes que representan sus núcleos de apoyo e interdependencia. La igualdad se basa en el principio de justicia en términos del derecho de todos lo individuos a recibir beneficios comparables considerando los casos similares de manera análoga y los casos diferentes con el reconocimiento de sus particularidades. La diversidad se refiere al respeto por las diferencias en valores, cultura, religión u orientación sexual, y al mismo tiempo se reconoce la universalidad de los derechos sexuales y reproductivos.

Pero no es suficiente su declaración formal, el ejercicio de derechos requiere además de condiciones posibilitadoras en términos de recursos y poder. Poder para tomar decisiones bien fundamentadas e informadas acerca de

${ }^{3}$ Que el derecho reproductivo se haya institucionalizado, dice Cervantes (1996), significa que su ejercicio depende de la lógica, la estructura, el proceder y la intencionalidad de las instituciones. la fertilidad, crianza de los hijos e hijas, salud ginecológica y actividad sexual; recursos para tomar estas decisiones de forma segura y efectiva, y condiciones para que esto ocurra (Correa y Petchesky, 1994). Es decir, requiere condiciones materiales (de infraestructura y servicios médicos humanitarios), políticas (de acceso a la educación, al salario, a la toma de decisiones), ético-culturales (tolerancia, respeto a la diversidad y reconocimiento de las mujeres como agentes morales), personales (empoderamiento y conciencia de derechos) y de relación (vínculos amorosos, relaciones solidarias, redes de apoyo) que equilibren las asimetrías en el acceso a poderes y recursos.

Por todo esto, no es casual que para las feministas el tema de los derechos reproductivos cristalice de muchas maneras toda la cuestión de los derechos humanos sobre sus propios cuerpos, en la medida en que sobre los cuerpos de las mujeres recae la disputa política del orden moral, de las políticas de población y las consideraciones mercadotécnicas presentes en las tecnologías reproductivas y anticonceptivas. En el área de la reproducción es donde los cuerpos de las mujeres difieren más significativamente de los hombres, porque estos procesos viven simbólicamente en el cuerpo de las mujeres y biológicamente son inseparables de sus probabilidades de enfermar o morir, pero además porque en los intentos oficiales de establecer el control sobre los cuerpos de las mujeres están en juego los intereses patriarcales y los capitalistas.

La batalla internacional por el cuerpo de las mujeres ha implicado una estrategia diferente para las mujeres de los países industrializados y para las mujeres de los países pobres. Mientras por un lado se trata de desalentar a las mujeres pobres del hemisferio sur a no reproducirse (para no criar más pobres que algún día podrían llegar a reclamar una participación de la riqueza que se les ha robado); por otro lado, a las mujeres de clase media, sobre todo las del norte, se las alienta a procrear porque incrementan la demanda de consumo que rige la acumulación del capital. El manejo oficial de los programas de población, al enfatizar la planificación familiar como estrategia en las políticas de desarrollo, ha representado más una transgresión que un intento de fortalecer la autonomía de las mujeres. Centrarse en la sobrepoblación como causa del subdesarrollo ha sido una manera de tender un velo 
sobre la distribución desigual de la riqueza por parte del sistema mundial y sobre la explotación inhumana de la fuerza de trabajo (Kabeer, 1998). Conculcados por la religión, acotados por las políticas poblacionales y disputados en los espacios de la intimidad, los derechos de las mujeres sobre su cuerpo y sus opciones reproductivas son un dique a los excesos de los micro y macropoderes, por ello resultan centrales para su sentimiento de individualidad y para definir un proyecto autónomo de vida. Las enormes dificultades que ello enfrenta tienen como base el foco de poder que representa el cuerpo como terreno de estos derechos.

Éste es el punto clave cuando se aborda el tema de los derechos reproductivos y los hombres, porque en su caso el cuerpo no es motivo de disputa ni mueren por evitar un embarazo ni las prácticas sexuales forzadas tienen efectos reproductivos en sus cuerpos, y además pueden

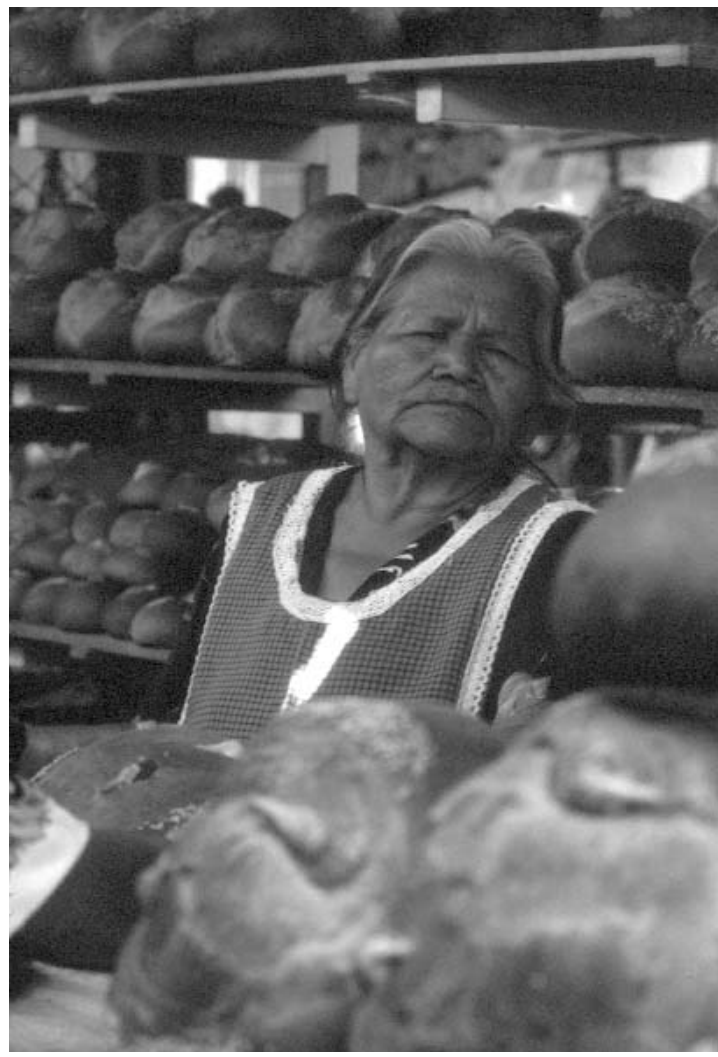

De la serie: Mercados hecho a mano, 2000 / Roxana Acevedo evadir las consecuencias de sus prácticas sexuales, no así las mujeres. Pero no es sólo el cuerpo anatómico lo que está en juego sino la historia inscrita en el cuerpo. Ellos como grupo y como individuos participan en el mantenimiento de este orden social y se benefician voluntaria o involuntariamente de él. Por ejemplo, en las familias organizadas patriarcalmente los miembros masculinos mayores cosechan los beneficios de familias numerosas, mientras que las mujeres en edad reproductiva cargan con los costos en forma de altas tasas de morbilidad y mortalidad materna. También la industria farmacéutica y los políticas de planificación familiar han concentrado sus esfuerzos en programas dirigidos a las mujeres, pese a que los métodos masculinos suelen ser más baratos, más seguros y más simples. Incluso las nociones de riesgo y seguridad se aplican a la probabilidad de embarazo asociado a fallas de los anticonceptivos y no con los efectos que estos tienen en la salud de las mujeres. Finalmente, pese a que hombres y mujeres son copartícipes en la reproducción, los riesgos y responsabilidades, tanto de la anticoncepción como del embarazo, aborto, parto y cuidado de la prole, recaen predominantemente en las mujeres. ${ }^{4}$

En gran medida tanto las fallas en las políticas públicas como los factores estructurales del orden genérico han contribuido a mantener esta situación, pero también las prácticas cotidianas de los hombres mismos y los beneficios que obtienen de la desigual distribución de poderes y recursos. Por esto no es de extrañarse que los esfuerzos por modificar el panorama de la salud reproductiva hayan destacado el papel de los hombres como obstáculos o facilitadores de los derechos reproductivos de las mujeres. No obstante, esto no significa negar a los hombres necesidades e intereses propios. De acuerdo con documentos de Naciones Unidas, la participación masculina vinculada a la salud reproductiva es un término "sombrilla" para abarcar los diferentes caminos en los cuales los hombres se relacionan con los problemas y los programas de salud reproductiva. Por un lado se

\footnotetext{
${ }^{4}$ Actualmente sólo $7.3 \%$ de los varones utiliza algún método anticonceptivo (Conapo, 2000).
} 
considera la forma en que los hombres aceptan y brindan apoyo a las necesidades, elecciones y derechos en salud reproductiva de sus compañeras, y por el otro, se trata de analizar el propio comportamiento sexual y reproductivo de los hombres (FASyDR-FNUAP, 1998). Con ello se pretende disminuir los riesgos en la salud de las mujeres, aumentar la participación de los varones en el cuidado de su propia salud y promover relaciones más equitativas y solidarias entre unos y otras.

\section{LOS HOMBRESY LOS DERECHOS REPRODUCTIVOS DE LAS MUJERES}

En el esfuerzo por mejorar la salud reproductiva y el bienestar de las mujeres, los niños y los hombres mismos, se hizo cada vez más evidente que no era posible avanzar sin incorporar a los hombres a los programas y políticas públicas relacionadas con la reproducción y la sexualidad. Las mujeres podían reclamar sus derechos frente al Estado, los gobiernos podían instrumentar políticas públicas, pero mientras no se politizara la vida privada y se equilibraran las relaciones de poder en los espacios de la intimidad, los programas seguirían fracasando puesto que las prácticas, actitudes y decisiones de los hombres tienen un efecto determinante en la salud de las mujeres.

Los hombres tienen más parejas sexuales (hetero, homo y bisexuales), inician más tempranamente su vida sexual, tienen relaciones extramaritales con más frecuencia, tienen más prácticas desprotegidas, son asintomáticos respecto a ciertos agentes infecciosos como el virus de papiloma humano y mantienen una vida reproductiva durante más años. ${ }^{5}$ Todo ello pone en riesgo a las mujeres de contraer enfermedades sexualmente transmisibles o de infectarse de VIH/sida, aumenta sus probabilidades de enfermar de cáncer cérvico-uterino, de vivir embarazos no deseados y de procrear en condiciones de riesgo. A su vez, la violencia física, sexual y emocional que viven

${ }^{5}$ Los diversos estudios sobre comportamiento sexual en México dan cuenta de ello; la Encuesta Nacional de Juventud (2000) y la Encuesta Metropolitana sobre Actitudes y Prácticas Sexuales (De la Peña, 2001) aportan datos concretos al respecto. las mujeres, ejercida en su mayor parte por los hombres, limita sus posibilidades de tomar decisiones que les permitan protegerse de contagios o embarazos no deseados. ${ }^{6}$ Por lo general, los hombres se involucran en la sexualidad y la reproducción desde una posición de control y dominio asumido por ellos como natural y compartido muchas veces por sus parejas. Sus intereses y deseos son cruciales en las decisiones de las mujeres sobre el aborto, el uso de métodos anticonceptivos y el número de hijos. Además, la falta de apoyo y responsabilidad de los hombres ante un aborto inducido, aumenta el riesgo de morbilidad y mortalidad en las mujeres y las coloca en una situación de mayor vulnerabilidad física y emocional. $^{7}$

No obstante, los hombres también facilitan el ejercicio de los derechos reproductivos de las mujeres. Cuando reconocen a sus compañeras como agentes morales capaces de tomar decisiones, apoyan las decisiones de ellas, comparten responsabilidades y promueven condiciones que facilitan el ejercicio de los derechos de sus parejas, la salud reproductiva de las mujeres y sus familias tiende a mejorar. De hecho señala HERA (1998), la participación responsable de los hombres fortalece a la sociedad en su conjunto.

Que los hombres actúen de una u otra manera no es un asunto de voluntad personal, no existe una malquerencia explícita en sus prácticas ni su conducta opera siempre en el plano de las intenciones conscientes, ellos como varones forman parte de una sociedad donde ocupan una posición de privilegio y desde esa posición perciben el mundo y se relacionan con las mujeres. Es, como dice Bourdieu (2000), desde la objetividad de las estructuras sociales y la subjetividad de los esquemas cognitivos que se construye la dominación. Esto es, las estructuras

\footnotetext{
${ }^{6}$ Una de cada dos mujeres requiere el permiso del esposo para protegerse contra un posible embarazo, mientras que en zonas rurales una de cuatro mujeres no usan ninguna medida anticonceptiva porque se los prohibe el esposo (Conapo, 2000).

${ }^{7}$ Sobre la forma en que las prácticas y actitudes de los hombres afectan la salud de las mujeres existen múltiples estudios, pero los trabajos pioneros de Liguori (1989) sobre el contagio de sida en mujeres, el conjunto de investigaciones reseñadas por Alatorre (2000) y los datos sobre la participación de los hombres en el aborto presentadas por Guevara (1998 y 1999) y por Tolbert, Ehrenfeld y Lamas (1996) son contundentes en ese sentido.
} 
de dominación son el producto de un trabajo continuo de reproducción al que contribuyen los agentes singulares (entre los que están los hombres con armas, la violencia física y la violencia simbólica), las instituciones como la familia, Iglesia, escuela, Estado y las mujeres mismas como reproductoras de un orden que las limita en todos los espacios sociales.

La objetividad del orden social se imprime insensiblemente en el orden de los cuerpos mediante los esquemas de percepción y apreciación que lleva a aceptar como evidente, natural y obvias las prescripciones más arbitrarias. La estructura impone sus coerciones tanto a quienes se benefician de ella como a quienes sufren sus consecuencias, no tanto por las sanciones institucionales sino porque engendra prácticas perfectamente ajustadas a ese orden y por tanto percibidas y valoradas por quien las lleva a cabo, y también por los demás, como justas, correctas, hábiles y adecuadas. Cada cual encuentra en el comportamiento de sus iguales la ratificación y legitimación de su propio comportamiento que, por ser fruto de las mismas condiciones de existencia y unos mismos condicionamientos, produce comportamientos adaptados a las condiciones objetivas. El habitus como sistema de disposiciones a ser y hacer, es una potencialidad, un deseo de ser que, en cierto modo trata de crear las condiciones de su realización y por lo tanto, de imponer las condiciones más favorables para la realización de las urgencias, los gustos, los afectos (Bourdieu, 1999).

Así, cuando un hombre y una mujer establecen relaciones en los espacios de la sexualidad erótica y reproductiva, lo hacen desde sus respectivas posiciones sociales y sus específicas condiciones vitales, toda la estructura social está presente en el núcleo de la interacción. Las imposiciones, acuerdos, pactos o posibilidades de negociación no ocurren en un vacío social, están determinados por el conjunto de capitales culturales y simbólicos con que cuente cada uno/a y por los poderes estructurales y coyunturales que entran en juego en un momento determinado. En general, las asimetrías de poder son la constante cuando se negocia en los terrenos de la sexualidad y la reproducción, y suelen ser los hombres los que deciden qué se negocia y qué no entra en la negociación. Los poderes de las mujeres dependen en mucho de su esta- tuto conyugal, de la calidad del vínculo amoroso y de sus propias posibilidades de autonomía.

Por todo esto es que se ha enfatizado el carácter empoderador de los derechos reproductivos en el caso de las mujeres a fin de reequilibrar las relaciones de poder. Por eso mismo resulta inadecuado hablar de los hombres "como seres que se reproducen" no sólo porque se niega el hecho biológico de que no existe ni remotamente la posibilidad de que un hombre se reproduzca sin que participe el cuerpo de una mujer, sino además porque se borra de un plumazo el sentido ético del concepto de responsabilidad ${ }^{8}$ que debiera ser al fin $\mathrm{y}$ al cabo el principio rector de las relaciones humanas, además de que éste es indisociable del concepto de derechos. Por esto también resulta inadmisible que, en una traducción mecánica de las categorías aplicadas a las mujeres, se pretenda identificar los derechos reproductivos de los hombres haciendo alusión a aquello que "les desagrada o los incomoda"9 sin tomar en cuenta la posición que ellos ocupan en la red de relaciones sociales. ${ }^{10}$ Baste un botón de muestra en la voz misma de los hombres sobre una situación que le desagrada en una experiencia de aborto inducido:

...mira ella me dijo que se cuidaba y a la mera hora me sa-
le con que está embarazada y que era"nuestro" problema,
entonces yo le dije "fíjate que no" (...) si hubiera sido una
mujer con un menor nivel cultural o que ella no tuviera cla-
ridad de lo que estaba haciendo, bueno, pero así no. Ella no
tenía por qué haberme metido en este lío. Finalmente deci-
dió que era algo que no quería vivir, pero que el responsable

\footnotetext{
${ }^{8}$ La responsabilidad supone siempre el reconocimiento de la existencia del "otro" puesto que implica considerar las consecuencias que tendrán las acciones y decisiones propias en otra u otras personas (Sánchez Vázquez, 1982). Más aun que en el terreno reproductivo no se trata de un otro generalizado y abstracto, sino un otro concreto para quien se tienen sentimientos y con quien se ha compartido experiencias eróticas, amorosas o se ha vivido episodios de violencia.

${ }^{9}$ Véase el artículo de Juan Guillermo Figueroa, "Varones, reproducción y derechos. ¿Podemos combinar estos términos?”, en Desacatos 6 de esta revista, página 162.

${ }^{10}$ En la presentación del proyecto sobre la forma en que las mujeres se apoderan de la noción de los derechos reproductivos, Rosalind Petchesky (1999) señala que al socializar aquellas experiencias que les parecen tristes, desagradables o violentas, las mujeres van desarrollando mecanismos para identificar sus derechos reproductivos. Esta idea se aplica mecánicamente a los hombres sin considerar las diferencias biológicas, sociales y simbólicas entre ambos.
} 
de eso era "yo" porque la había embarazado. ¡Debí haberla mandado a la chingada! [¿Tú que hiciste una vez que se tomó la decisión del aborto?] Nada, si era su asunto que ella lo resolviera [¿Cómo te sentiste?] Yo tenía otras cosas que hacer, no pensaba mucho en eso (Guevara, 2001).

La voz de este hombre que no ejerce violencia física y "sólo" se desatiende del asunto, muestra que aquello que les incomoda como varones aún cuando puede estar vinculado a sus derechos reproductivos, se encuentra directamente relacionado con sus posibilidades de ejercicio del poder. También muestra los límites que marca el cuerpo a los poderes de uno y otra, mientras él puede "hacer otras cosas" ella tiene que enfrentar las consecuencias, le guste o no.

Esto no significa que la dominación sea un destino ineludible ni que los derechos de unos y otras representen una suma cero. Los hombres, como todos los seres

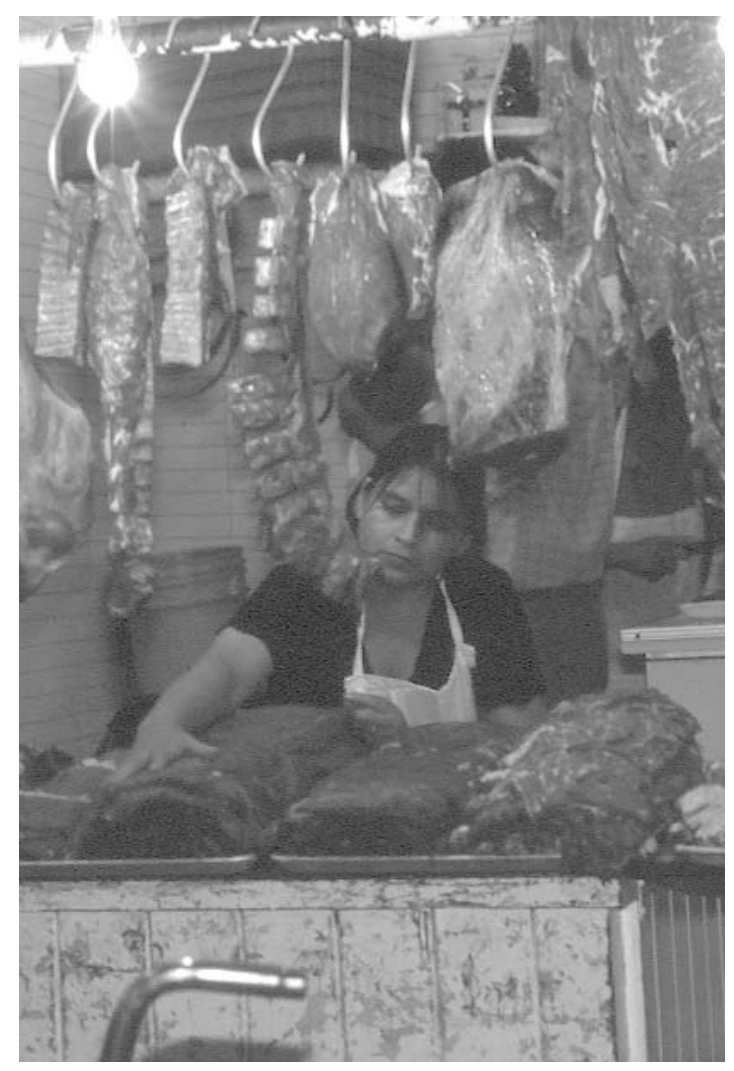

De la serie: Mercados hecho a mano, 2002 / Roxana Acevedo humanos, responden a sus condiciones y si éstas cambian se ven obligados a modificar sus prácticas. No existe un instinto infalible de dominación o ejercicio del poder, y los esquemas de percepción, de acción y de relación con que actúan los hombres no son siempre coherentes y tienen diversos grados de contradicción, incertidumbre e incomodidad con las posiciones que ocupan. Además, las transformaciones estructurales han modificado las instituciones mismas y el lugar que ocupan los individuos dentro de ellas, esto ha obligado a un reacomodo de poderes en los espacios público y privado e impulsado distintas formas de relación.

Así, existen situaciones para los hombres en que la salud y la vida de sus compañeras es tan importante como su propia salud y su propia vida, situaciones en las que la responsabilidad compartida disminuye las asimetrías y situaciones en las que las mujeres pueden contar con más poderes y recursos para protegerse y negociar. En estos casos los derechos reproductivos de las mujeres se colocan en primer plano, y muchos hombres agradecen que así sea. Ellos como varones no viven los procesos reproductivos en sus cuerpos, pero en ocasiones el lazo que los une con su compañera puede tener una enorme fuerza simbólica. Es la calidad de este vínculo lo que, algunas veces, hace inseparables los derechos de unos y otras. ${ }^{11}$ De igual manera, cuando la acción social de los hombres, como individuos y como entidades colectivas, se comprometen con un práctica política orientada a reequilibrar los poderes y a revisar su participación en el mantenimiento de las estructuras de dominación, se pueden crear espacios de solidaridad, de alianzas y complicidades con las mujeres que permite enfrentar de manera conjunta los poderes del Estado, del mercado o de los fundamentalismos religiosos.

En ocasiones se olvida que el concepto de hombres o masculinidad no es una mera referencia demográfica a

\footnotetext{
${ }^{11}$ Por ejemplo, en un estudio realizado con hombres urbanos y profesionistas, que habían vivido cuando menos un aborto inducido, se encontró que la experiencia del aborto fue difícil para la mayoría de ellos, pero lo fue más cuando mantenían un fuerte vínculo cercano con su compañera. En algunos casos, la falta de condiciones seguras para la realización de un aborto fue considerada por ellos como un atentado a su propia seguridad y bienestar (Guevara, 2001).
} 


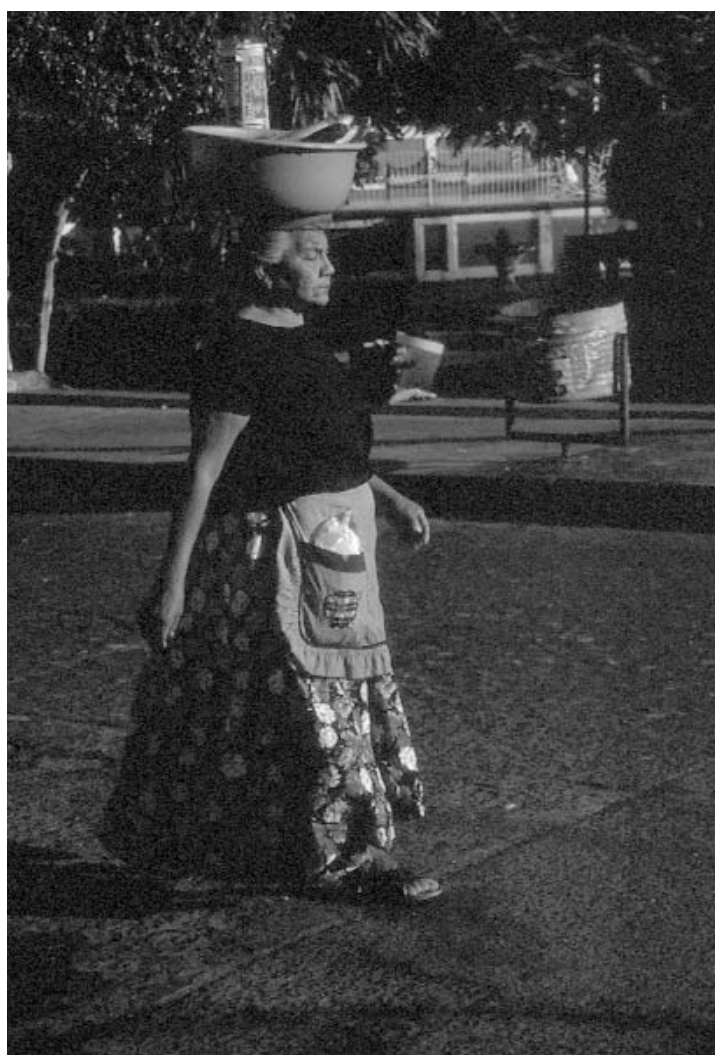

De la serie: Mercados hecho a mano, 2000 / Roxana Acevedo

una población ni se trata sólo de estereotipos sino que, en el marco de la perspectiva de género, representa una categoría de análisis con un profundo sentido político. Por ello no debemos preguntarnos tanto por los atributos personales como por el tipo de configuración social que hace posible ciertas posiciones de poder y ciertas prácticas políticas. En el caso de los derechos reproductivos es fundamental definir a los hombres y mujeres por su posición dentro de la red de relaciones sociales, porque las reivindicaciones políticas de género tienen sentido no sobre la idea de que las capacidades innatas de unos u otras han sido obstruidas sino, como señala Alcoff (1989), porque la posición que ocupan las mujeres dentro de la red carece de poder y movilidad y es necesario modificarla. Precisamente éste es el punto medular en el ámbito de los derechos reproductivos, pues la noción de derechos parte de una perspectiva emancipatoria que se mueve en distintos frentes de disputa teórica y política. Es en estos frentes donde habría que buscar el sentido de los derechos reproductivos de los hombres.

\section{LOS DERECHOS REPRODUCTIVOS DE LOS HOMBRES, LAS OTRAS INTERROGANTES}

Dos elementos son fundamentales en la conceptualización de los derechos reproductivos: la premisa de indivisibilidad de los derechos humanos y la noción de un ambiente favorable para el ejercicio de estos derechos (Correa, 1996). Con base en ello se puede decir que los hombres, en su mayoría, tienen garantizados sus derechos políticos, económicos y sociales y por tanto, sus derechos reproductivos, además de que en general cuentan con las condiciones, en términos de poderes y recursos, para el ejercicio de estos derechos. No obstante, las relaciones de género están inmersas en otras formas de desigualdad social y los poderes están distribuidos en distintos campos sociales, de manera que muchos hombres ya sea por su condición de clase, de etnia, de edad o de orientación sexual, también se encuentran en posiciones de desventaja para el ejercicio de sus derechos.

Es frecuente que en razón de su edad, de su residencia en una zona rural o marginal, que por su condición de migrantes o de indígenas, o en razón de los sesgos de género presentes en las políticas públicas, muchos hombres no tengan acceso a la información y a la educación sexual, a los medios para protegerse de embarazos no deseados o de contagio de ATS/sida, y tampoco a servicios de salud para la detección y atención a sus procesos reproductivos. Más aún, existen denuncias sobre esterilizaciones forzadas en indígenas quienes han sido vasectomizados con engaños y en flagrante violación a la normatividad establecida en las instituciones de salud. ${ }^{12} \mathrm{~A}$

\footnotetext{
${ }^{12}$ El caso más difundido es el de 16 mixtecos a quienes se practicó la vasectomía con engaños, bajo la promesa de entregarles dinero y con amenazas de excluirlos del Progresa y Procampo. Ellos interpusieron una denuncia ante la Comisión de Derechos Humanos de Guerrero, y ante la falta de respuesta la CNDH ha turnado la recomendación 18/2001. De todo ello da cuenta un reciente reportaje de Víctor Ballinas (2001) en La Jornada.
} 
pesar de ello, se puede decir que en estos casos no es tanto su condición de hombre lo que limita u obstruye el ejercicio de sus derechos reproductivos, sino su pertenencia a sectores sociales que han sido excluidos de los derechos más elementales.

En cambio existen otros casos, donde precisamente su condición de hombre es el núcleo de la discusión respecto a las posibilidades de reconocimiento a sus derechos reproductivos. Es el caso de los varones homosexuales, de quienes se encuentran infectados con VIH/sida y los hombres con discapacidad mental. En estos sectores, el punto central es que al negarles el estatuto de hombres se les niega también el estatuto de personas y la posibilidad de tener derechos. Estos presupuestos no son explícitamente enunciados sino que se encuentran ocultos en los estigmas y en una moral social que justifica con los argumentos más falaces la discriminación y la exclusión de que son objeto. Incluso, las violaciones no sólo se circunscriben al acceso a la información o a los servicios de salud, sino al más elemental derecho de procrear o no. Cuando se trata de hombres infectados o de varones con discapacitadad mental, existen argumentos éticos en el sentido de que el ejercicio de su derecho a procrear puede afectar derechos fundamentales de terceros (de la pareja o hijos/as), pero en el caso de los varones homosexuales ni siquiera existen argumentos éticos, es sólo el peso de la intolerancia, de una moral social discriminatoria y una jurisprudencia homófoba que los desconoce como personas y les niega sus posibilidades de existir con derechos plenos.

Ante esta situación es difícil entender por qué razón en los debates sobre los derechos reproductivos de los hombres hay una ausencia notable de reflexión sobre estos temas, $\mathrm{y}$ a veces ni siquiera una mención a estos casos. $\mathrm{Ni}$ la violación a los derechos reproductivos de los indígenas, ni las formas que los hombres homosexuales negocian o no con las mujeres con quienes procrean, ni los debates legislativos sobre los derechos reproductivos en las parejas homosexuales, nada de esto ocupa a quienes escriben sobre el tema. Al parecer los atentados a la dignidad y los derechos humanos de los varones que se encuentran en condiciones estructurales de desventaja, no es una preocupación central en la agenda política y la reflexión teórica que realizan los hombres sobre el tema. ¿Por qué?

Hasta ahora, el debate que han impulsado los varones en el plano nacional e internacional sobre sus derechos reproductivos, ha sucedido principalmente en tres frentes de disputa política: a] Los que se vinculan a las políticas públicas en salud reproductiva por las omisiones y fallas que presentan para incorporar a los hombres en sus programas y propuestas; b] Las que se sitúan en el ámbito de las condiciones laborales que limitan su participación, responsabilidad y disfrute en sus procesos reproductivos; y c] las disputas que se han dado desde la academia o las organizaciones civiles por la titularidad de los derechos reproductivos de los hombres frente a las mujeres. ${ }^{13}$

En el primer caso se trata de una reivindicación colectiva hacia los gobiernos por parte de amplios sectores de la población interesados en promover la participación responsable de los hombres en el ámbito de la salud reproductiva y el ejercicio de los derechos reproductivos. Se trata de demandas orientadas al diseño de servicios de salud reproductiva para los hombres, del entrenamiento a prestadores de servicios para una adecuada atención a sus necesidades, estrategias de educación a la comunidad, recursos de comunicación e información dirigidas a los hombres a fin de promover el cuidado en su salud, así como su participación responsable en la prevención de enfermedades de transmisión sexual, el uso de medidas anticonceptivas y el apoyo a sus parejas en la planificación familiar, el embarazo y parto. Investigadores como Wegner et al. (1998) señalan que aun cuando se asume casi automáticamente que los hombres no están interesados en participar de manera responsable, los estudios consultados por estos autores muestran que existe un genuino interés tanto por parte de los hombres como de

\footnotetext{
${ }^{13}$ En nuestro país es frecuente que los debates académicos sobre los derechos reproductivos de los hombres no se expresen estas disputas de manera abierta, sino que se encuentren encubiertas bajo versiones "neutras" del orden genérico. Es decir, se vacía de contenido político las reivindicaciones de las mujeres y se abordan las relaciones de género como si la desigualdad fuera producto de un sistema social abstracto en el que los hombres no tienen responsabilidad alguna y en el que son tan "oprimidos" como las mujeres.
} 
sus compañeras de ser tomados en cuenta en las políticas públicas. En este marco, de relación entre los individuos frente al Estado, los derechos reproductivos de los hombres tienen el mismo sentido que en el caso de las mujeres: la posibilidad de tomar decisiones autónomas, asumir responsabilidades y superar necesidades.

Respecto a las demandas sobre las condiciones laborales, el frente de disputa se encuentra en las relaciones entre el individuo y las instancias de regulación social como el mercado y el Estado que limitan el derecho de los hombres a participar y apoyar los procesos de gestación, parto y crianza de los hijos/as. En este caso la reivindicación de los derechos se dirige a obtener respuestas a necesidades no reconocidas de los hombres e impulsar los cambios legales que ello requiere.

En cuanto a la disputa que enfrentan los hombres por la titularidad de sus derechos frente a las mujeres, el panorama se modifica sustancialmente porque ya no se trata de la relación entre los individuos y las instancias de regulación social, la disputa se sitúa en el terreno de los derechos de un individuo frente a otro y de los mecanismo institucionales, simbólicos y materiales con que cuenta cada uno para defender sus derechos. Aquí el concepto de los derechos reproductivos en los hombres pierde su sentido de empoderamiento y el objetivo político apunta en contra de las mujeres.

En los dos primeros casos existe un amplio consenso entre los grupos feministas, organismos multilaterales y grupos de hombres por apoyar estas demandas. Gloria Careaga (1999) señala que en el proceso de cabildeo del Programa de Acción de El Cairo, la adhesión de los grupos de hombres de la Unión Europea reforzó la demanda de que la perspectiva de género fuera contemplada como un capítulo especial. La propuesta que ellos plantearon, dice Careaga, se insertaba de manera muy precisa en modificar las relaciones de inequidad y en que reconocía esta inequidad como consecuencia de las diferencias biológicas y las limitaciones sociales impuestas hacia las mujeres en los procesos reproductivos. Esto es, su propuesta se sustentaba en el reconocimiento de la responsabilidad paterna en la crianza de la descendencia y en la necesidad de reconocer su papel en la reproducción, donde incluían la revisión de las leyes laborales que les permitieran asumir estas responsabilidades por igual con las mujeres, pero sobre todo revisar su participación en las estructuras de poder y comprometerse con impulsar la participación de las mujeres en todas las esferas de la vida.

Estas demandas de los hombres son perfectamente coincidentes con los proyectos políticos del feminismo y sobre este ámbito no existe contraposición de intereses con las mujeres, hay un objetivo común y un esfuerzo conjunto por modificar las estructuras de inequidad. Desafortunadamente no ocurre así en el tercer frente de disputa que han impulsado algunos hombres. Aquí se ubican desde los movimientos más conservadores como los promovidos por los grupos Pro Vida o los grupos parlamentarios del PAN, hasta algunas propuestas desde la academia o las organizaciones civiles sobre los derechos reproductivos de los varones. ${ }^{14}$ Éstos últimos aun cuando ocupan el espectro político opuesto, formalmente apoyan las reivindicaciones feministas y sostienen el principio de la libre elección, al ignorar las dimensiones éticas y políticas en el análisis de los derechos reproductivos, abren la puerta a las posturas más regresivas y permiten reforzar las estructuras de dominación que los feminismos debilitaron.

En estos casos, el discurso de los derechos, de la igualdad y la democracia, sirven para disputar a las mujeres espacios de legitimación duramente conseguidos, porque como bien señalaba Weber (1997), la dominación descansa en el discurso de legitimidad y hacia allá apuntan estos discursos. En la medida en que la posición de los varones está siendo desafiada por el discurso de los derechos de las mujeres, se utilizan las herramientas teóricas creadas por las mujeres mismas para recomponer la posición de los hombres bajo el principio de la igualdad. De forma velada se trata de demostrar que los hombres

\footnotetext{
${ }^{14}$ Esta postura es muy clara en el caso de los escritos sobre el tema de Juan Guillermo Figueroa como el artículo publicado por esta revista en su número 6 del año 2001, pero también ha sido frecuente escuchar estas consideraciones entre quienes desde la academia o las organizaciones civiles, incluidas algunas mujeres, trabajan el tema de masculinidad y salud reproductiva. Con frecuencia se confunde igualdad con equidad y se habla de las "paternidades forzadas" como muestra de las "violaciones" a los derechos reproductivos de los hombres bajo argumentos totalmente carentes de sustento teórico.
} 


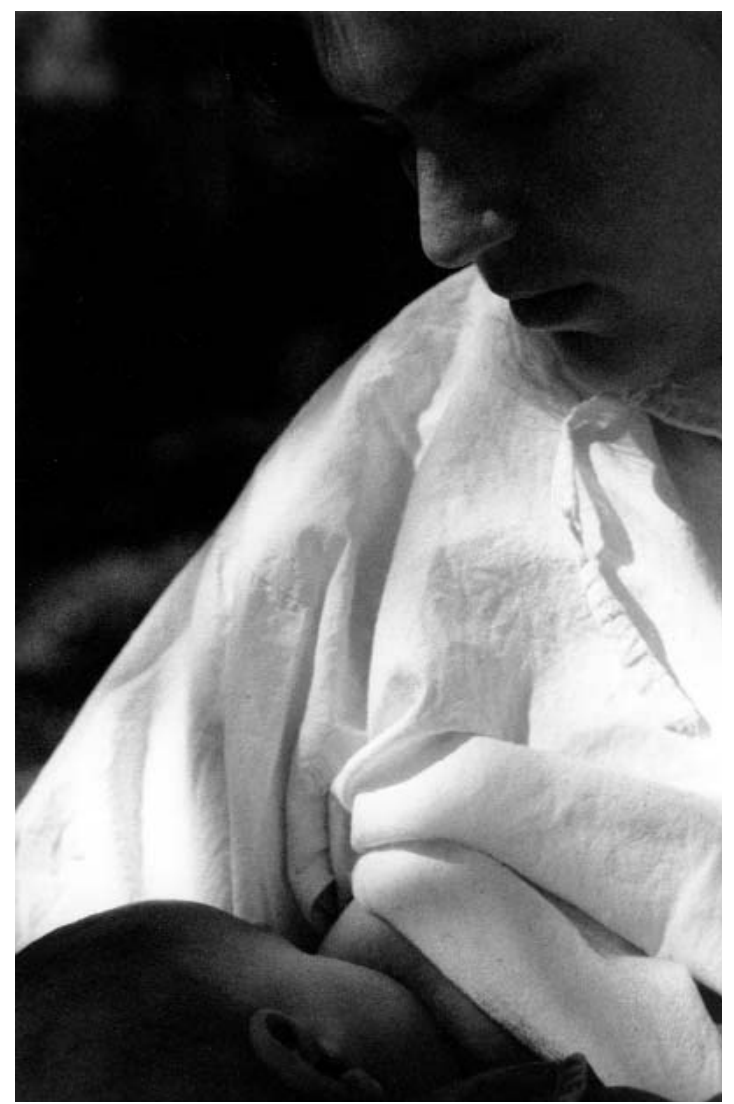

De la serie: Entre dos te quiero más, 2003 / Roxana Acevedo

son los grandes “olvidados" en el discurso de los derechos reproductivos, pero los argumentos utilizados más parecen orientados a competir con las mujeres y arrebatarles espacios de legitimación que a construir relaciones solidarias o desmantelar las estructuras de dominación. Especialmente parecen dirigidos a recuperar el protagonismo de los hombres al irrumpir en espacios que permiten a las mujeres desafiar los poderes masculinos. Todo ello en un contexto de endurecimiento hacia cualquier forma de autodeterminación y bajo un discurso que facilita interpretar los actos afirmativos de las mujeres como formas de transgresión, de agresión o desafío a lo que se consideran los "derechos" de los hombres.

Es claro que desde la ética y la filosofía moral, la libertad de elección y el ejercicio de derechos siempre involucra a otros actores sociales que también son sujetos de derecho; éstos pueden ser otros individuos, la familia, la colectividad o el Estado. Cuando existe incompatibilidad entre los derechos de unos y otros, existen distintas modalidades de negociación de los derechos y las responsabilidades que se resuelven básicamente por tres caminos: mediante la conciliación de intereses de todas las partes en una propuesta que permita llegar a orientaciones comunes; mediante al apelación a los principios éticos que permita dirimir las diferencias y otorgar legitimación a quienes cuentan con menos poder, o mediante la imposición de alguno de los actores sobre el otro u otros con base en discursos que legitiman su poder.

En cualquiera de los casos, los poderes de cada uno de los actores definen el marco de negociación en función de las opciones que les permiten los sistemas de desigualdad en que se hayan inmersos. Más aun cuando se trata del ámbito reproductivo donde el cuerpo de las mujeres es el terreno en disputa y cuando no puede haber equivalente en la otra parte. Como señala Macklin (1996), el hecho de que los procesos reproductivos se gesten en los cuerpos de las mujeres, les confiere a ellas el derecho de autodeterminación sobre sus cuerpos y tal derecho no tiene contraparte o paralelo en los hombres. No puede apelarse a la equivalencia en este plano, puesto que la ética señala que se debe tratar de manera semejante casos semejantes y de manera diferente los casos diferentes, por ello cuando existen divergencias el principio de libertad individual demanda que prevalezca el derecho de las mujeres. El dilema ético consiste precisamente en cómo hacer compatible este principio de libertad individual con el derecho de los hombres a vivir su paternidad sin presiones. No obstante, todo derecho supone responsabilidades y el punto de partida en cualquier negociación debe ser el hecho incontrovertible de que los procesos reproductivos ocurren en el cuerpo de las mujeres.

Sin embargo, estas consideraciones son omitidas en el debate sobre el tema, se habla del derecho de los hombres a participar como si ellos no participaran en estas decisiones. De hecho, existen múltiples evidencias de que participan de muy variadas maneras, ${ }^{15}$ en algunos casos

${ }^{15} \mathrm{El}$ caso del aborto es un buen ejemplo. Tolbert, Ehrenfeld y Lamas (1996) muestran que en América Latina entre un 30 y $40 \%$ de las 
respetan y apoyan las decisiones de su compañera; en otros, negocian; y en otros más se imponen. La disputa de derechos en este caso adquiere características muy específicas porque ocurre en los espacios privados, entre dos personas con derechos y obligaciones no siempre coincidentes, con importantes diferenciales de poder, pero unidos por relaciones eróticas, amorosas o institucionales. Y como toda pugna de derechos supone un problema de relación, la relación misma se pone a prueba y se redefine en estas disputas.

Las formas de conexión son vitales para entender los procesos que dan lugar al ejercicio de los derechos en los espacios de la intimidad. Es desde los vínculos que se definen los poderes y los mecanismos de negociación, se establece el tipo de responsabilidad y se fijan los límites de cada uno al interior de la relación. Cuando hombres y mujeres negocian en el terreno de la reproducción no establecen sólo acuerdos coyunturales, de hecho negocian su proyecto de vida, su proyecto de pareja, sus expectativas de paternidad o maternidad y su propia relación. ${ }^{16}$ Pero estas negociaciones no se establecen en un plano de paridad, sino que forman parte de ciertas reglas del juego que legitiman formas veladas de ejercicio del poder presentes en las distintas formas de relación amorosa. ${ }^{17}$

A diferencia de las mujeres, los hombres tienen muchas más posibilidades de defender sus derechos frente a sus compañeras sexuales y definir su proyecto de vida de acuerdo con sus intereses. Ellos tienen más probabilidades de evitar la paternidad no deseada o de tener acceso a la paternidad si así lo desean, pues la posición que ocupan en la sociedad les otorga más poderes dentro y fuera de la relación les "autoriza" para ejercer sus derechos en

mujeres que abortan exponen que la razón por la que no desean el hijo es el rechazo del varón al embarazo.

16 Por ello no es posible circunscribir la negociación en el terreno de la reproducción a la negociación de la coitalidad, puesto que en las relaciones de la intimidad se negocia mucho más que una práctica sexual.

17 Por ejemplo, la evidencia empírica muestra (Guevara, 1999) que cuando los hombres viven un embarazo no deseado en una relación ocasional o extramarital, la continuación del embarazo ni siquiera entra en la negociación, si acaso se negocia el apoyo material que ellos pueden ofrecer para realizar un aborto, pero no su paternidad. Esas son las reglas del juego que ellos establecieron y no están dispuestos a modificar. todos los terrenos y no les exige requisitos de castidad para tomar decisiones. Si llegaran a vivir prácticas sexuales forzadas, éstas no tienen efectos reproductivos en sus cuerpos y cuando asumen su responsabilidad en la anticoncepción, tienen mayores garantías de que sus prácticas sexuales no tendrán consecuencias reproductivas.

Por ello, cuando se habla de los derechos reproductivos desde el feminismo se trata de destacar la estrecha articulación que existe entre las disputas micropolíticas de la vida cotidiana y las posiciones que ocupa cada uno en las estructuras e instituciones sociales. Por esta misma razón, la libertad de elección se incluye en el campo más amplio del ejercicio de la ciudadanía, que se entiende no sólo como garantías legales y beneficios del Estado sino como un espacio de transformación de las estructuras sociales y las relaciones de género. Este es el piso en que se han movido las reivindicaciones asociadas a los derechos reproductivos de las mujeres, pero, ¿qué derechos afirmativos defienden los hombres cuando hablan de sus derechos reproductivos?, ¿dónde se encuentra el reequilibrio de poder respecto a las mujeres?, ¿qué papel desempeña el cuerpo de las mujeres ante una disputa de derechos?, ¿de qué manera vinculan la interdependencia con la autodeterminación?, ¿qué factores estructurales limitan sus derechos más allá de la clase la etnia o la preferencia sexual?, ¿cómo se pueden desmantelar las estructuras de dominación sin reconocer las asimetrías de poder?, ¿dónde está el compromiso de los varones por revisar su participación en el mantenimiento de estas estructuras?

No debiéramos olvidar que los derechos reproductivos dependen de condiciones aún no disponibles para la mayoría de los seres humanos, y por tanto, las posibilidades de ejercicio de estos derechos se encuentran inequitativamente distribuidas en razón de distintos ejes de desigualdad social. El avance de los fundamentalismos religiosos, el creciente poder político obtenido por la derecha en nuestro país y el papel cada vez mayor de las corporaciones trasnacionales en los espacios de la reproducción humana, hacen prever condiciones más adversas para el ejercicio de los derechos y apuntan a restringir cada vez más los espacios que hacen posible la libertad de elección. Por esto, sin una clara definición política y un riguroso trabajo teórico, los objetivos de justicia y empodera- 
miento presentes en la noción de derechos reproductivos se perderán en un horizonte de indefiniciones que sólo servirá para refuncionalizar el discurso de la opresión.

\section{Bibliografía}

Alatorre, Javier, 2000, "Incorporación de los hombres a las acciones de salud sexual y reproductiva. Revisión de las investigaciones, programas y acciones", mecanograma, PUEG, UNAM.

Alcoff, Linda, 1989, "Feminismo cultural versus pos-estructuralismo: la crisis de la identidad en la teoría feminista", en Feminaria, núm. 4: 1-18.

Ballinas, Victor, 2001, "Investigar esterilización a mixtecos, insiste la CNDH”, en La Jornada, 14 de septiembre, p. 37.

Bellinghausen, Hermann, 2000, "Se incrementa represión en Chiapas. La procuraduría estatal y Pro Vida allanan clínica para mujeres en aras del derecho a la vida", en $L a$ Jornada, 20 de octubre, p. 14.

Bourdieu, Pierre, 1999, Meditaciones pascalianas, Anagrama, Barcelona.

—_, 2000, La dominación masculina, Anagrama, Barcelona.

Careaga, Gloria, 1999, "Políticas internacionales respecto a la participación del hombre en la salud sexual y reproductiva", Encuentro Nacional Los Varones Frente a la Salud Sexual y Reproductiva, Secretaría de Salud-Conapo-Pueg-AVSC International, México.

Cervantes, Alejandro, 1996, "De mujeres médicos y burócratas: Políticas de población y derechos humanos en México", en Careaga G., Figueroa J.G. y Mejía M.C., Ética y salud reproductiva, Miguel Ángel Porrúa-PUEG, México.

Conapo, 2000, La fecundidad y la planificación familiar, Consejo Nacional de Población, México.

Correa, Sonia, 1996, "Salud reproductiva, género y sexualidad. Legitimación y nuevas interrogantes", Seminario Internacional sobre Avances en Salud Reproductiva y Sexualidad, Colmex, México.

— y Petchesky, Rosalind, 1994, "Reproductive and Sexual Rights: a Feminist Perspective”, en Sen, G., A. Germaine y L. Chen, Populations Policies Re-Considered: Health, Empowerment and Rights, Harvard School of Public Health, Boston.

De la Peña, Ricardo, 2001, "El nuevo orden sexual", Encuesta Metropolitana sobre Actitudes y Prácticas Sexuales, revista Nexos, agosto, núm. 284: 58-74.

Durand, Teresa y María Alicia Gutierrez, 1998, "Tras las huellas de un porvenir incierto: del aborto a los derechos sexuales y reproductivos", en AEPA-CEDES-CENEP, Avances en investigación social en salud reproductiva y sexualidad, Buenos Aires, Argentina.

Encuesta Nacional de Juventud, 2000, Resultados preliminares, 2000, Secretaría de Educación Pública, Instituto Mexicano de la Juventud, Centro de Investigaciones y Estudios sobre Juventud, México.

FASyDR-FNUAP, 1998, Participación y responsabilidad masculina en la sexualidad, reproducción y crianza, Foro Abierto de Salud y Derechos Reproductivos, Santiago de Chile.

Guevara R., Elsa, 1998, "Amor y pareja en la responsabilidad de los hombres frente al aborto", en AEPA-CEDES-CENEP, Avances en investigación social en salud reproductiva y sexualidad, Buenos Aires, Argentina.

_, 1999 , "La corresponsabilidad ética de los varones frente al aborto", ponencia presentada en las. Jornadas Australes Interdisciplinarias sobre Mujer y Desarrollo, Facultad de Medicina, Universidad Austral de Chile, Valdivia, Chile.

— , 2001, "La experiencia del aborto en los hombres y los derechos reproductivos", en Revista de Estudios de Género La Ventana, núm. 14, vol. II: 242-266, Universidad de Guadalajara, México.

HERA, 1998, "Men's role and responsability for sexual and reproductive rights and health", en Action Sheets, Health Empowerment Rights and Accountability, Nueva York.

Kabeer, Naila, 1998, Realidades trastocadas: las jerarquías de género en el pensamiento del desarrollo, México: PUEGPaidós.

Liguori, Ana Luisa, 1989, "Políticas en salud: mujer y sida", en Tapia E. y P. Mercado, Mujeres y politicas públicas, Fundación Friedrich Ebert, México.

Macklin, Ruth, 1996, "Ética y reproducción humana: Perspectivas internacionales", en Careaga G., Figueroa J.G. y Mejía M.C., Ética y salud reproductiva, Miguel Ángel Porrúa-PUEG, México.

Mejía Ma. Consuelo, 1999, "Cairo +5. ¿El consenso de El Cairo en riesgo?”, en Conciencia Latinoamericana, vol. XI, núm. 2: 2-4.

Sánchez Vázquez, Adolofo, 1982, Ética, Grijalbo, México.

Tolbert, K. Ehernfeld, N. y Lamas, M., 1996, "El aborto en México: un fenómeno escondido en proceso de descubrimiento", en Langer, A. y Tobert, K., Mujer: sexualidad y salud reproductiva en México, Population Council, Oficina Regional para América Latina, México.

Weber, Max, 1997, Ensayos sobre metodología sociológica, Amorrortu, Argentina.

Wegner, Mary, Evelyn Landry, David Wilkinson y Joanne Tzanis, 1998, "Men as partners in reproductive health: From issues to action", en International Family Planning Perspectives, vol. 24, núm. 1: 38-42. 